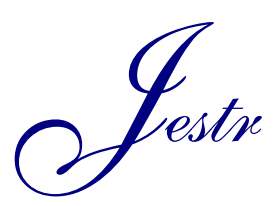

\title{
Formation Damage and Wellbore Stability of Soft Mudstone Subjected to Thermal- Hydraulic-Mechanical Loading
}

\author{
Mingyu Pang ${ }^{1,2}$, Gao Xu ${ }^{1}$, Feng Sun ${ }^{1}$, Shifeng Xue ${ }^{1, *}$ and Yalong Wang ${ }^{3}$ \\ ${ }^{1}$ College of Pipeline and Civil Engineering, China University of Petroleum, Qingdao, 266580, China \\ ${ }^{2}$ Sinopec Research Institute of Safety Engineering, Qingdao, 415504, China \\ ${ }^{3}$ Petro-Geotech Incorparated, Calgary, 2P3 E5, Canada
}

Received 2 June 2019; Accepted 14 August 2019

\begin{abstract}
The wellbore stability of soft mudstone formation is strongly affected by thermal and hydraulic loadings due to its low strength and high plasticity under high-pressure and high-temperature conditions. A nonlinear coupled thermalhydraulic-mechanical (THM) model was proposed to evaluate the influence of temperature and fluid factors on wellbore stability located in deep and complex soft mudstone formations. The evolution of near-wellbore formation strength and permeability was quantitatively described by introducing a plastic damage variable in the THM model. The influence of mud pressure and temperature on the stress and plastic damage distribution near the wellbore zone was analyzed using the THM model. The accuracy of the models was verified by comparing the radial/hoop stress results with those from classical thermopore elastic theory. Results show that the plastic failure of the near-wellbore zone and wellbore stability are mainly controlled by the coupling effects of thermal and hydraulic deformations of the soft mudstone. Specifically, the size of the damage zone is enlarged by $2.7 \mathrm{~mm}$ when the mud temperature increases by $40{ }^{\circ} \mathrm{C}$. By contrast, the damage scale is reduced by $1.7 \mathrm{~mm}$ as the temperature decrease by $40{ }^{\circ} \mathrm{C}$ due to the compaction effects in the nearwellbore region. The mechanical behavior of the soft mudstone wellbore is time dependent, that is, with the increase in time, the damage gradually extends to the periphery of the wellbore after the plastic equilibrium state near the wellbore is reached. This study provides a THM modelling framework for understanding the mechanism of soft mudstone wellbore instability and formulating corresponding preventive measures.
\end{abstract}

Keywords: Soft mudstone formation, Wellbore stability, Thermal-Hydraulic-Mechanical Coupling, Plastic damage

\section{Introduction}

With the exploration and development of deep oil/gas and geothermal resources, wellbore damage failure and gas leakage accidents increase rapidly under high-pressure and high-temperature (HPHT) conditions of complex formation $[1,2]$. These problems contain complicated characteristics, such as absorption, expansion, and nonlinear deformation, of soft mudstone formation in deep formation. In addition, the damage softening phenomena in the temperature and fluid environments, which deteriorate the deformation and stress distribution near the wellbore region and accelerate its instability and damage, result in a significant increase in drilling costs. Analysis of wellbore stability under thermal and hydraulic loading conditions is becoming increasingly prominent during drilling [3].

At present, the wellbore instability and failure mechanism in complicated formation environments have attracted considerable attention from scholars. Efforts have been exerted on the development of elastic and inelastic formations and pore flow-related models. However, the traditional wellbore stability models have only been applied to soft mudstone formations because only the mechanical strength or deformation mechanism of the wellbore structure

*E-mail address: xuesf@126.com

ISSN: 1791-2377 @ 2019 School of Science, IHU. All rights reserved.

doi:10.25103/iestr.124.11 is considered. Hence, developing a wellbore stability mechanical model coupled with wellbore temperature, flow, and nonlinear formation deformation is necessary.

The local stress field in wellbore is gradually reconstructed during drilling, leading to increased highstress concentration. This phenomenon further causes plasticity deformation, damage, and failure near the wellbore zone, especially for the sensitive soft mudstone formations in the later stage of production and operation of oil/gas wells $[4,5]$.

Generally, the stress and deformation distribution and the damage degree of near-wellbore zones are used as the key mechanical parameters for the quantitative evaluation of wellbore stability. Limited studies have been conducted on the effects of thermal-hydraulic coupling, plastic deformation, damage, and failure to investigate the mechanism of wellbore stability during drilling. As a result, integrating the thermal-hydraulic-mechanical (THM) coupling effects is crucial in the wellbore structure analysis.

In this study, a coupled THM model of mudstone wellbore stability under thermal and hydraulic loadings was developed. The stress distribution pattern and damage evolution process during drilling were quantitatively analyzed using nonlinear finite element method (FEM) of the multiphysical field. This study aimed to predict the range of the near-wellbore plastic damage zone under thermalhydraulic loads accurately and provide a reference for the 
stability control technology of soft mudstone wellbore structures.

\section{State of the Art}

The THM coupling that occurs in porous medium formations is extremely complex [6]. Formation damage and wellbore instability caused by the changes of pore fluid pressure and temperature have always been a leading research topic in oilfield engineering. On the basis of the geological environment, mechanical behavior, and loading conditions, the study of wellbore stability in multiphysical field coupling is generally classified as THM, thermalmechanical (TM), hydraulic-mechanical (HM), or seepagedeformation coupling.

McTigue [7] derived the analytical solution of the stress distribution in wellbore on the basis of the elasticity of TM coupling theory. This model assumed that the formation is an elastic porous material and that the pore permeability and mechanical property parameters are constant with time, which is relatively different from those of engineering practice. The thermoelastic stress near the wellbore induced by the change in drilling mud temperature $\left(150{ }^{\circ} \mathrm{C}\right)$ was studied numerically by Maury et al. [7]. Their results suggested that the thermal effects of the wellbore temperature cannot be ignored. Li Jingyuan et al. [8] considered the HM effects, Mohr-Coulomb criterion, and damage and softening of formation; they also analyzed the elastoplastic stress distribution near the wellbore but ignored the TM coupling effect during drilling. The THM coupling of elastic medium theory and the models for wellbore stability were reviewed by Roegers [9]. However, the effects of initial stress during drilling were not discussed. Ghassemi et al. [10] proposed a nonisothermal elastic THM coupling model for shale reservoirs but did not incorporate the quantitative analysis of the damage of formations near the wellbore into their method. A coupled mathematical model of elastic THM of geotechnical materials was established by Zhang Wei [11] and Jia Shanpo et al. [12]. The secondary distributions of the temperature, seepage, and stress fields near the wellbore zone during drilling were quantitatively analyzed, excluding the nonlinear deformation of formation. Kanfar [13] studied the THM coupled model of anisotropic formation and obtained the influence of the anisotropic heat transfer characteristics on wellbore stress. Zhang Pengwei et al. [14] established the HM coupling model of wellbore stability in nonlinear soft mudstone formations on the basis of plastic-damage theory and analyzed the plastic softening and HM coupling effects during drilling. In their work, the temperature effects were ignored. Gray [3, 15] studied the cake effects of drilling mud on wellbore stress, plastic damage, plastic-strain softening and permeability correction. However, the thermal effect of the drilling mud temperature was also ignored. $\mathrm{Wu} \mathrm{Yi}$ et al. [16] analyzed the thermal stress under circulating low-temperature drilling fluid. Their results showed that decreasing the mud temperature reduces the collapse of the formation and improves the wellbore stability. However, the seepage near the wellbore zone during drilling was ignored.

On the basis of the field data, the reconstruction or reformation of in situ stress field during drilling is an important starting point for evaluating wellbore stability in the entire production life cycle. During drilling, the upper part of the wellbore is heated by circulating mud, whereas the bottom of the wellbore is heated only when the mud circulation is stopped. This nonuniform heating of the upper and lower wellbore structures will cause expansion or shrinkage deformation [7]. In addition, few studies have been conducted to investigate the THM bidirectional coupling mechanism of soft mudstone formations thoroughly in the wellbore stability evaluation.

The evolution of near-wellbore formation strength and permeability was quantitatively described in this study by considering the plastic softening characteristics and introducing a plastic damage variable of soft mudstone under HPHT environment in the THM model. Apart from the factors of pore fluid seepage and wellbore temperature, this study focused on the drilling load/displacement boundary changes and the nonlinear softening of soft mudstone formations during the staged FEM analysis. Then, all THM coupling characteristics of the wellbore structure in mudstone formations were discussed, including stress distribution, damage evolution, and structure integrity.

The remainder of this study is organized as follows: Section 3 establishes the THM coupling numerical model of soft mudstone wellbore stability and presents the solution process of the numerical model. Section 4 compares the experimental and theoretical solutions of the wellbore thermopore elastic model to verify the accuracy and reliability of the numerical model. The evolution of wellbore stress and damage caused by time-dependent wellbore temperature, seepage characteristics during drilling, and the influence of mud temperature on wellbore stability is numerically analyzed. Finally, Section 5 summarizes the study and provides the main conclusions.

\section{Methodology}

\subsection{Mechanical constitutive equation of soft mudstone formations}

\subsubsection{Plastic softening model}

The elastoplastic strain softening model of soft mudstone was established based on the Drucker-Prager strength criterion. Since the formation failure is indeed a gradual damage process, the stress-strain curve of the whole process for the soft mudstone obtained from the experiments has various forms. Generally, in order to describe the intrinsic deformation characteristics, the stress-strain curve can be modeled as a simple form, as shown in Fig.1.

In Fig. 1, $\mathrm{AB}, \mathrm{BC}$, and $\mathrm{CD}$ represent the linear elastic response, strain softening, and residual stress phases, respectively. The post-peak $\mathrm{B}$ at the strain softening stage is the most important parameter, which affects the failure limitation level of soft mudstone. Beyond the post-peak value, the Drucker-Prager yield criterion is used to describe the plastic yield at the peak stress state for soft mudstone [17].

$F(\boldsymbol{\sigma}, c)=\sqrt{J_{2}(s(\boldsymbol{\sigma}))}+\eta p(\boldsymbol{\sigma})-\xi c$

where $p=\operatorname{tr}[\sigma] / 3$ is hydrostatic pressure, $J_{2}=\frac{1}{2} \boldsymbol{s}: \boldsymbol{s}$ is the second invariant of stress deviation, $\boldsymbol{s}=\boldsymbol{\sigma}-p(\boldsymbol{\sigma}) \boldsymbol{I}$ is the deviatoric stress tensor, and $\mathcal{C}$ is cohesive force. $\eta, \xi$ are variables related to the internal friction angle $\varphi$, respectively. 
$\eta=\frac{6 \sin \varphi(m)}{\sqrt{3}[3-\sin \varphi(m)]}, \quad \xi=\frac{6 \cos \varphi(m)}{\sqrt{3}[3-\sin \varphi(m)]}$

where $m$ is the softening parameter.

In the proposed constitutive model, it is assumed that the strength and the strain softening parameters are in the form of a piecewise linear function. $\zeta$ represents the cohesive force, $c$ and internal friction angle $\varphi$ of the D-P yield criterion, and the relationship between $\zeta$ and $\gamma$ can be expressed as follows:

$\zeta(\gamma)=\left\{\begin{array}{lr}\zeta_{p}-\left(\zeta_{p}-\zeta_{r}\right) \frac{\gamma}{\gamma^{*}}, & 0<\gamma<\gamma^{*} \\ \zeta_{r}, & \gamma \geq \gamma^{*}\end{array}\right.$

where $\zeta_{p}$ and $\zeta_{r}$ represent the intensity parameter at the peak and at the residual phase, respectively. $\gamma^{*}$ represents the critical point between softening stage $\mathrm{BC}$ and residual stress phase $\mathrm{CD}$.

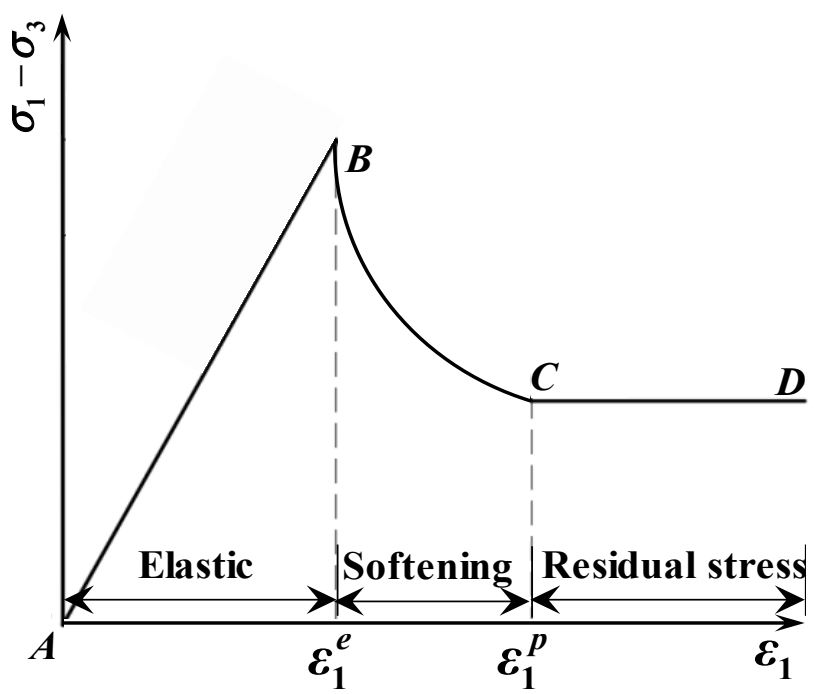

Fig. 1. Soft mudstone stress strain curve

\subsubsection{THM coupling model equation of soft mudstone}

The formation deformation of incremental format is expressed as [18].

$\{\Delta \sigma\}=[D]\{\Delta \varepsilon\}$

where $\{\Delta \sigma\}$ is the total stress increment, $\{\Delta \varepsilon\}$ is the strain increment, and $[\boldsymbol{D}]$ is the elastoplastic coefficients matrix.

In saturated porous media, the effective stress is introduced into Equation (4).

$\{\Delta \boldsymbol{\sigma}\}=\left[D^{\prime}\right]\{\Delta \boldsymbol{\varepsilon}\}+\Delta p_{f} \alpha\{m\}$

where $[D]^{\prime}$ is the effective elastoplastic coefficient matrix, $\{m\}^{\mathrm{T}}=\left\{\begin{array}{llllll}1 & 1 & 1 & 0 & 0 & 0\end{array}\right\}, \Delta p_{f}$ is the pore pressure change, and $\alpha$ is the Biot coefficient, respectively.

Under non-isothermal conditions, the total strain increment $\{\Delta \boldsymbol{\varepsilon}\}$ can be expressed as the sum of the mechanical strain increment $\left\{\Delta \boldsymbol{\varepsilon}_{\sigma}\right\}$ and the thermal strain increment $\left\{\Delta \varepsilon_{T}\right\}$.

$\{\Delta \boldsymbol{\varepsilon}\}=\left\{\Delta \boldsymbol{\varepsilon}_{\sigma}\right\}+\left\{\Delta \boldsymbol{\varepsilon}_{\boldsymbol{T}}\right\}$

where $\left\{\Delta \boldsymbol{\varepsilon}_{T}\right\}^{T}=\alpha_{T} \Delta T\{m\}, \quad \alpha_{\boldsymbol{T}}$ is the linear expansion coefficient of the formation skeleton, and $\Delta T$ is the temperature increase of the formation medium.

Combining Equations (5) and (6), the stress increment equation of the formation THM coupling is expressed as:

$\{\Delta \boldsymbol{\sigma}\}=\left[D^{\prime}\right]\{\Delta \boldsymbol{\varepsilon}\}-\left[D^{\prime}\right](\Delta T) \alpha_{T}\{m\}+\Delta p_{f} \alpha\{m\}$

Based on the mass conservation equation of pore fluid and Darcy's law, the fluid seepage equation is expressed as:

$\phi \beta_{w} \frac{\partial p}{\partial t}-\nabla \cdot\left[\frac{k}{\mu}\left(\nabla p+\rho_{f} g \nabla z\right)\right]+\nabla \cdot \frac{d \boldsymbol{u}}{d t}=f_{w}$

where $\phi$ is porosity, $\beta_{w}$ is the compression factor, $k$ is the permeability of the formation, $\mu$ is the viscosity of the fluid, $\rho_{f}$ is the fluid density, $\boldsymbol{u}$ is the formation deformation vector, and $f_{w}$ is the source item of fluid.

Energy conservation equation for soft mudstone matrix has the following form [19].

$(\rho c)_{t} \frac{\partial T}{\partial t}-k_{t} \nabla^{2} T-q_{t}=0$

where $(\rho c)_{t}=(1-\phi) c_{s} \rho_{s}+\phi c_{f} \rho_{f}$ represents the total heat capacity of the formation and fluid system, $k_{t}=\phi k_{f}+(1-\phi) k_{s}$ is the total thermal conductivity, $q_{t}$ is the internal heat source intensity, and $c_{s}, c_{f}, \rho_{s}, \rho_{f}, k_{s}, k_{f}$ represent the specific heat, density and thermal conductivity of the formation and fluid system, respectively.

A renew local permeability formula of formation is expressed as [20].

$\Omega=A_{0} e^{-\varepsilon_{p e} / a}+B_{0}$

$k=(1-\Omega) k_{m}+\Omega k_{\Omega}\left(1+\varepsilon_{v}\right)^{3}$

where $\Omega$ is the damage variable, $\varepsilon_{p e}$ is an equivalent plastic strain, $a$ is a material parameter and can be obtained experimentally, $A_{0}=1 /\left(\mathrm{e}^{-1 / a}-1\right), B_{0}=-1 /\left(\mathrm{e}^{-1 / a}-1\right)$ are the coefficients relate to $a, \varepsilon_{v}$ is the volumetric strain, and $k_{m}, k_{\Omega}$ is the permeability for non-damage and damage, respectively.

\subsection{FEM numerical model of wellbore stability}

\subsubsection{Geometric model}

Sheng Li oilfield (Shandong, China) was used as the study target zone. Fig. 2 shows the relevant data of wellbore temperature, fluid pressure, geostress, and boundary 
parameters. The stress, plastic deformation, and damage near the wellbore region under the THM coupling mechanism were explored. In view of the complexity of the actual wellbore structure, a 1/4 plane strain FEM model was established. The model size was $5 \mathrm{~m} \times 5 \mathrm{~m}$ and the wellbore radius was $0.1 \mathrm{~m}$.

The depth of wellbore formation is $1030 \mathrm{~m}$. According to the data of logging, the Young's modulus of soft mudstone is $16.0 \mathrm{GPa}$, Poisson's ratio is 0.25 , cohesion is $3.0 \mathrm{MPa}$ and internal friction angle is $40^{\circ}$. When the plastic damage limit is reached, the cohesion is reduced to $0.2 \mathrm{MPa}$. The temperature of the formation and mud of wellbore are $85^{\circ} \mathrm{C}$ and $45^{\circ} \mathrm{C}$, respectively. The pressure of the wellbore and formation are $12 \mathrm{MPa}$ and $8 \mathrm{MPa}$. All other parameters of the model, including thermal and hydrodynamic properties of the wellbore/formation, are shown in Table 1.

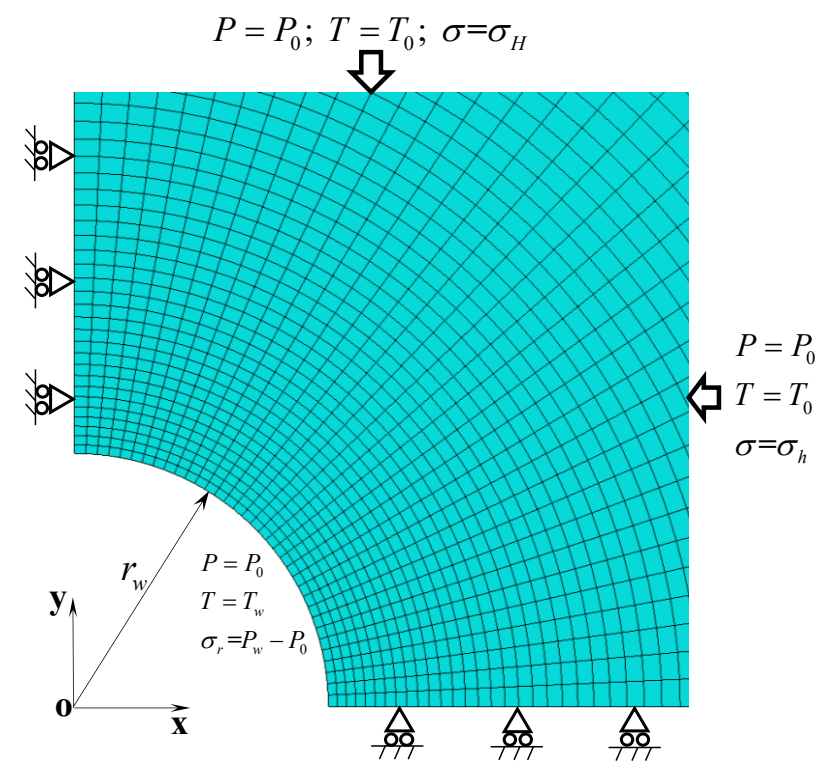

Fig. 2. FEM numerical model of wellbore stability

Table 1. The parameters of wellbore/formation

\begin{tabular}{l|c|c}
\hline Hydraulics & Permeability coefficient $(\mathrm{m} / \mathrm{s})$ & $1 \times 10^{-11}$ \\
& Pore ratio & 0.25 \\
\hline \multirow{3}{*}{ Thermodynamic } & Thermal Conductivity $\left(\mathrm{W} / \mathrm{m}^{\circ} \mathrm{C}\right)$ & 13 \\
& Specific heat capacity $\left(\mathrm{J} / \mathrm{kg}^{\circ} \mathrm{C}\right)$ & 18000 \\
& Thermal expansion coefficient $\left(1 /{ }^{\circ} \mathrm{C}\right)$ & $3 \times 10^{-5}$ \\
\hline
\end{tabular}

\subsubsection{Numerical simulation process}

The simulation scheme of the multiphysical process for soft mudstone wellbore stability was designed to accommodate the complex thermal loading at the wellbore, hydraulic seepage, and solid medium removal of wellbore during drilling. The three steps of the process were initial geostress equilibrium, drilling process, and THM coupling.

Initial geostress equilibrium. Before the drilling phase, the zero displacement of formation corresponds with a stabile stress field, namely, the initial equilibrium state of geostress or in situ stress. This geostress data from geological analysis is stored in an ODB file for subsequent dynamic calls.

Simulations of the drilling process. The birth-anddeath element in FEM is used to simulate the drilling or excavating removal process. When the elements inside the well hole are progressively killed, the corresponding mud pressure is simultaneously subjected to the inner wall of the wellbore.

THM-coupled process. The wellbore mud pressure, temperature, and horizontal principal stress loads were applied, and the THM response of the near-wellbore zone is simulated during drilling. In addition, the permeability of formation is updated dynamically using Equation (11) at each increment step.

The simulation scheme of the coupled THM models is designed to solve the temperature (T) and $\mathrm{HM}$ fields sequentially [11]. The T and HMs field are two independent systems that can be calculated separately. The HM coupling simulation includes the linearization of equations and increment optimization of loads. The, the $\mathrm{T}$ data are passed into the HM field as the loading to weaken the coupling between the T and HM fields at each incremental step.

\section{Result Analysis and Discussion}

\subsection{Model verification of thermopore elastic coupling}

The elastic THM coupling stress near the wellbore zone, including temperature, pore fluid pressure, elastic radial stress, and hoop stress, was analyzed to verify the validity of the model and method. During drilling, the pore pressure and formation temperature distributions were analyzed, as shown in Fig. 3.

A stable seepage process from wellbore to formation was assumed. The wellbore has a high pressure of $12 \mathrm{MPa}$, and the formation has a low pressure of $8 \mathrm{MPa}$. A reverse thermal conduction process from the formation to the wellbore was also showed in the Fig.3, where the temperature of the formation and wellbore are $85^{\circ} \mathrm{C}$ and $45^{\circ} \mathrm{C}$, respectively. In this process, the $\mathrm{TH}$ coupling effects were not considered, only the TM and HM coupling of the near wellbore were analyzed, respectively. Comparisons between the numerical results and the analytical solutions of WANG et al. [21] show a good agreement. At the normalized distance, the relative error is less than $5.6 \%$, indicating that the model is valid.

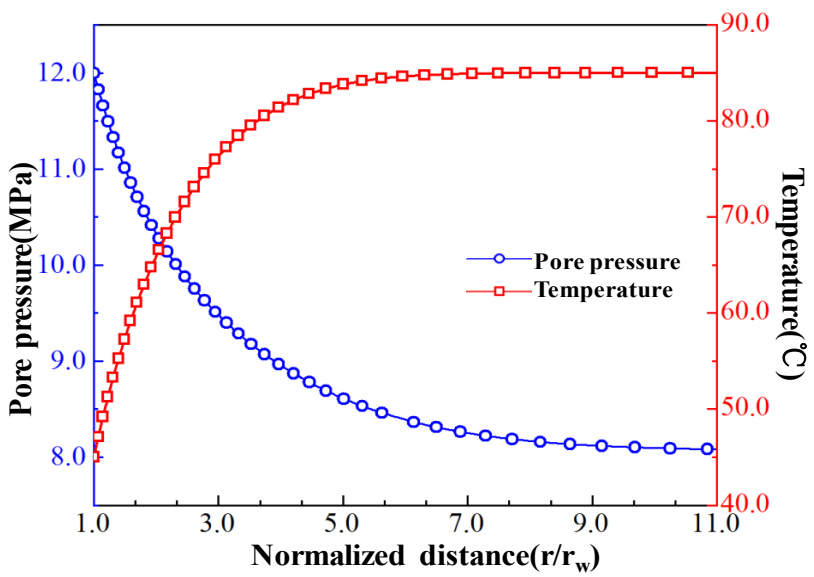

Fig. 3. The pore pressure and temperature plotted with the normalized distance

\subsection{THM coupling effects of nonlinear soft mudstone}

The THM coupling effects and their influence on the plastic deformation, stress, and damage of the near-wellbore zone were further analyzed. The nonlinear volumetric strain of the formation had an important effect on the permeability and pore structure, which were updated using Eqs. (10) and (11), respectively. Figs. 5(a) and 5(b) show the variations of temperature and pore pressure, respectively, with the normalized distance obtained at THM coupling durations of $0,0.3,2.8$, and $24 \mathrm{~h}$. At the initial moment, the wellbore and formation zones were isolated, and no heat conduction occurred between the wellbore and formation. Thus, the 
temperature field was uniform. With the drilling excavation, the hydraulic and thermal connections between wellbore and formation were set up. The wellbore wall temperature gradually decreased to the mud temperature by $45^{\circ} \mathrm{C}$ after $0.3 \mathrm{~h}$, and the temperature profile near the wellbore zone conformed to the typical heat diffusion pattern.

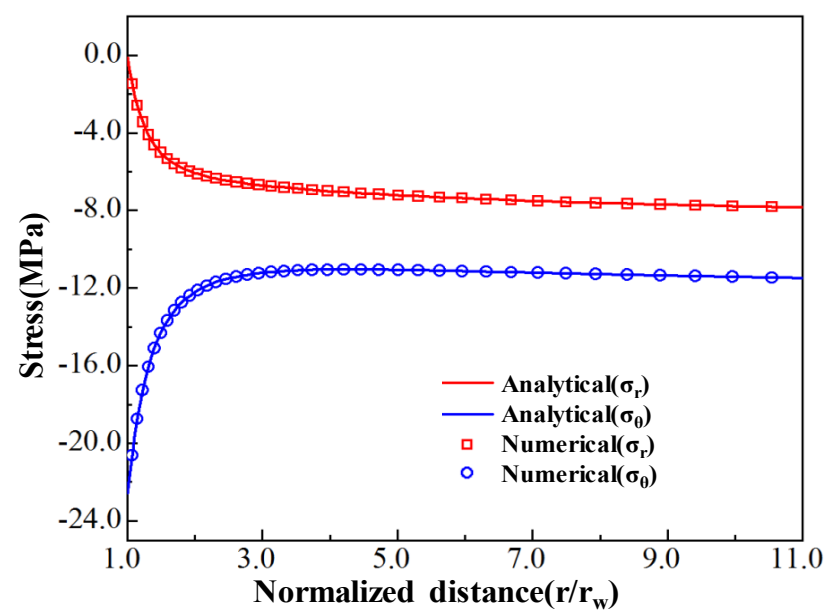

Fig. 4. Comparisons of numerical results with analytical solutions

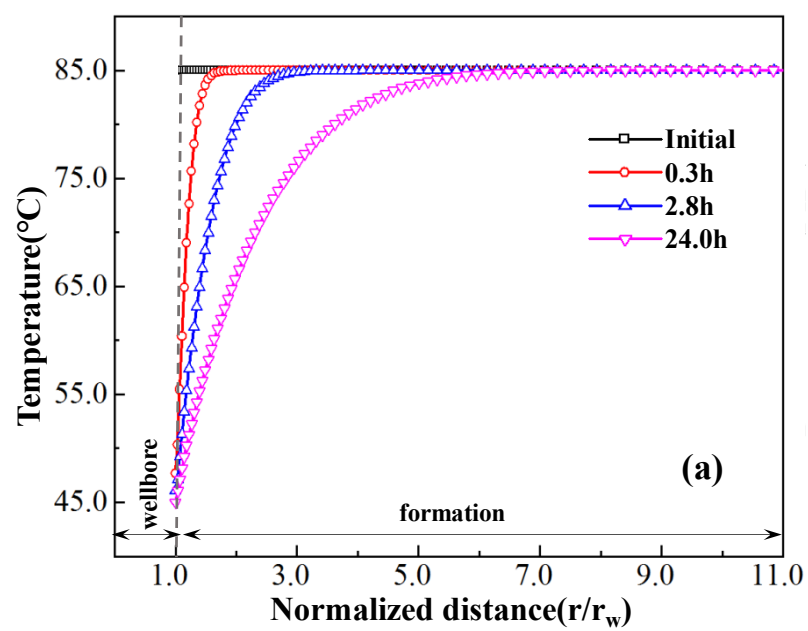

(a) Temperature

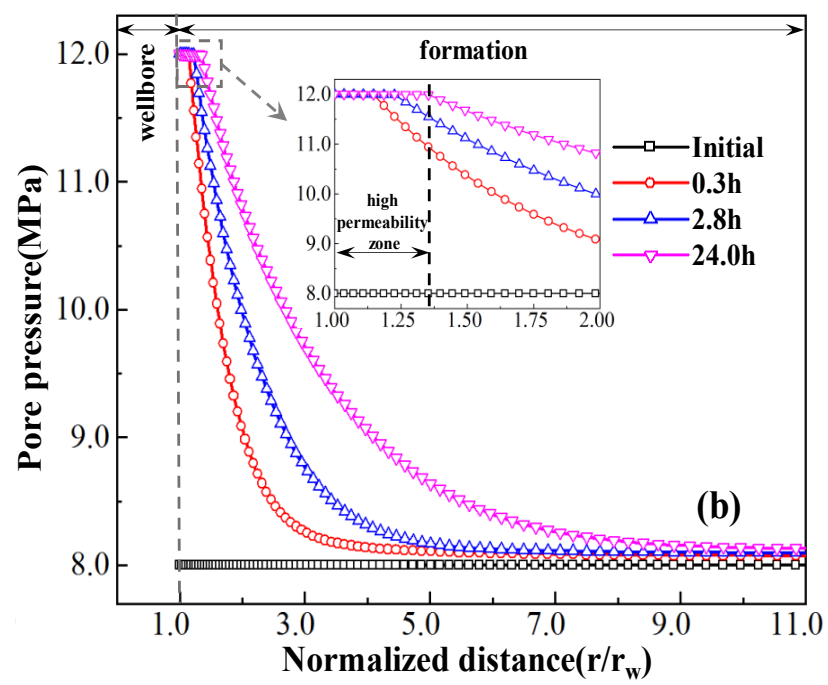

(b) pore pressure

Fig. 5. The variation of temperature with the normalized distance (at THM coupling duration of $0,0.3,2.8$ and $24 \mathrm{~h}$ )
The plastic damage zone is formed near the wellbore zone, which leads to an increase of the porosity and permeability. The pore pressure distribution evolves from the initial uniform steady state to the funnel shape of pressure drop. After $24 \mathrm{~h}$, a localized high permeability zone appears in the range of 1.3 times of wellbore radius, as shown in Fig. 5(b). The distribution pattern of pore pressure in this high permeability zone will further enhance the development of plastic damage, which has a negative effect on the wellbore stability. In our experiments, we have observed that PSO-net not only gives better modularity values, but also yields meaningful community structures. Figs. 4 and 5 show the ground truths and the obtained clustering results for the karate and dolphin networks.

On the basis of the variation of temperature and pore pressure near the abovementioned wellbore zone, the THM coupling effects on the wellbore stability was quantitatively evaluated by the change in stress distribution. The results in Fig. 7 showed that the effective radial stress at the wellbore was close to zero and the variation range was small under THM coupling. With the increase in coupling duration, the effective radial compressive stress near the wellbore region was gradually decreased.

In comparison with the elastic THM coupling stress distribution shown in Fig. 4, the distribution of effective hoop stress near the wellbore zone shown in Fig. 6(b) exhibited a clear difference. The position of the maximum hoop effective stress migrated from the wellbore $\left(r / r_{w}=1.0\right)$ to the periphery of the wellbore $\left(r / r_{w} \geq 1.2\right)$. With the increase in coupling time, this position moved gradually away from the wellbore, thereby reflecting the stress balance adjustment and plastic zone development in soft mudstone formations. Therefore, the effective hoop stress near the wellbore was the main control parameter for wellbore structure stability [18].

Under the plastic damage and THM coupling effects, the effective hoop stress at the wellbore was adjusted from the elasticity (-22.5 MPa) to the plasticity $(-7.2 \mathrm{MPa})$, as shown in Fig. 6(b). The final effective radial stress reduced to zero, which could reduce the local stress concentration at the wellbore, as shown in Fig 6(a). The plastic deformation zone, which was approximately 5.0 times that of the radius near the wellbore zone, was formed progressively due to the THM coupling effects.

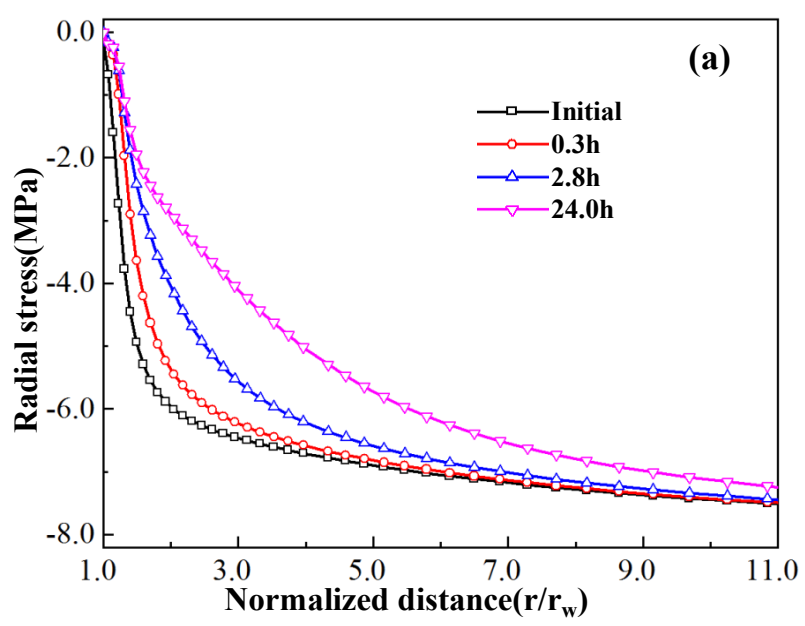

(a) Effective radial stress 


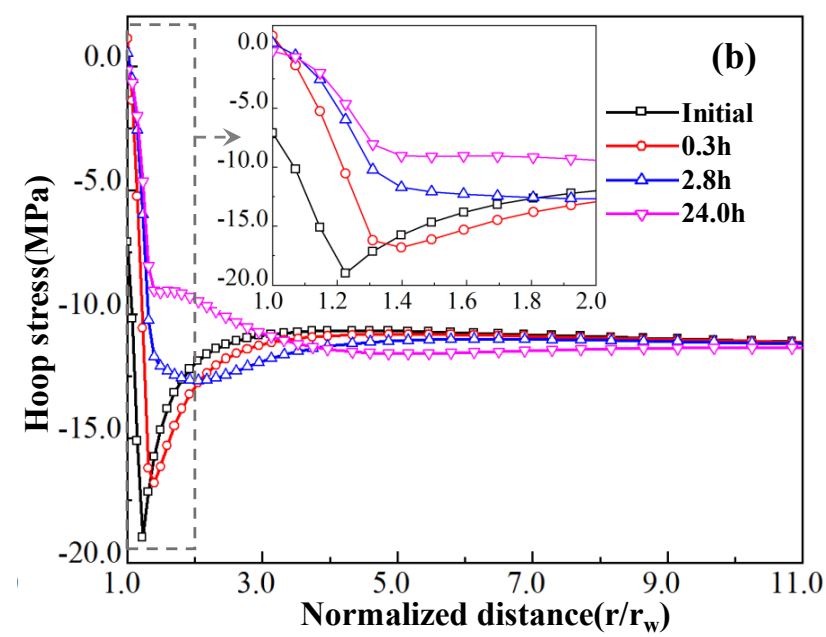

(b) Effective hoop stress

Fig. 6. The effective stress near the wellbore plotted with the normalized distance

\subsection{Plastic deformation and damage near the wellbore} zone

As previously mentioned, when the plastic strain near the wellbore zone accumulates to a certain extent, the soft mudstone formation will be damaged. In this study, the wellbore damage was characterized by a critical equivalent plastic strain, which was set to $1.0 \%$.

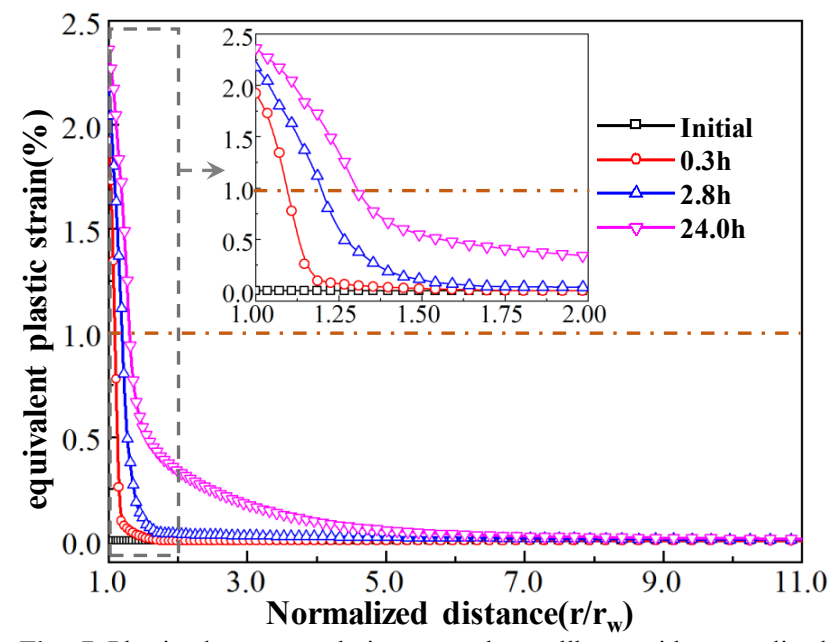

Fig. 7 Plastic damage evolution near the wellbore with normalized distance

Fig. 7 shows the plastic damage evolution of the mudstone formation near the wellbore under the THM coupling effects. At $0.3 \mathrm{~h}$, the plasticity occurred outside the wellbore along the minimum horizontal stress, but it still maintained a high hoop stress concentration. At this moment, the plastic damage value reached $1.9 \%$, exceeding the critical value of $1.0 \%$, thereby resulting in damage, as shown in Fig. 8(a). At $24 \mathrm{~h}$, the plastic damage zone expanded to the periphery of the wellbore and finally reached the position of the wellbore $\left(r / r_{w}=1.3\right)$, as shown in Fig. 8(b).

From the plastic deformation and stress distribution pattern near the wellbore mentioned above, the zone with a range of $1.3 \leq r / r_{w} \leq 5.0$ is a plastic deformation zone, and the elastic deformation is dominated in the zone with the range of $r / r_{w}>5.0$, see Fig. 8(b). The plastic damage degree and scale near the wellbore zone have important reference value for subsequent completion and wellbore stability control.

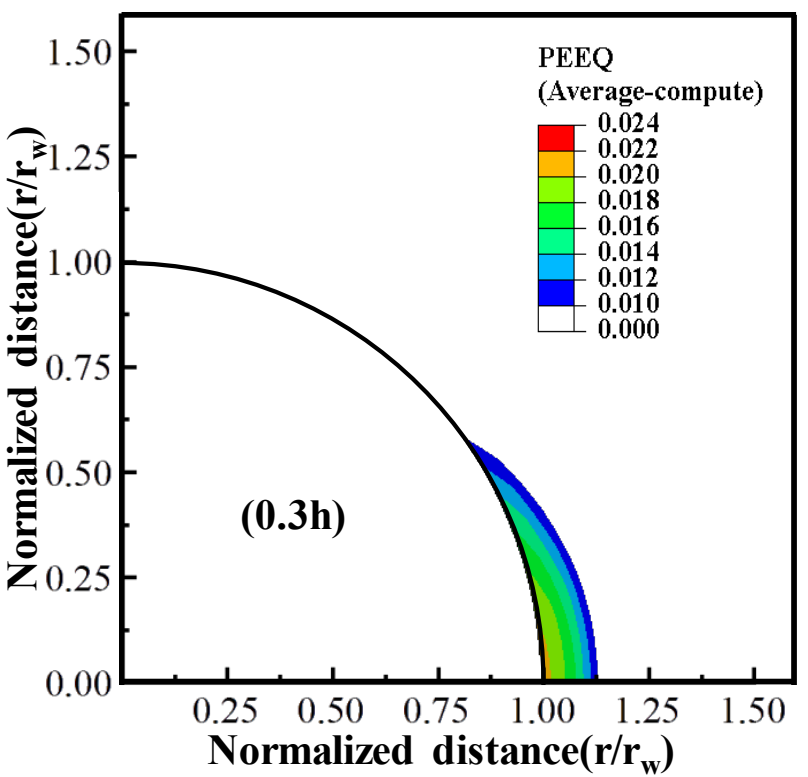

(a) THM coupling duration of $0.3 \mathrm{~h}$

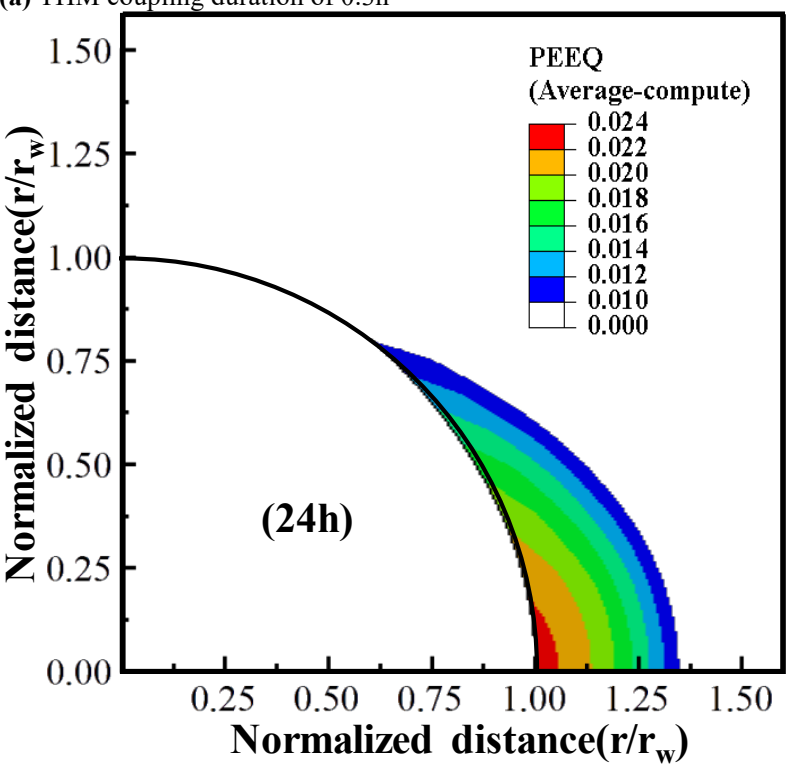

(b) THM coupling duration of $24 \mathrm{~h}$

Fig. 8. Plastic damage evolution of soft mudstone wellbore

\subsection{Influence of temperature on wellbore stability}

Temperature and thermal stress are impact factors affecting the plastic damage near the wellbore zone. The stress distribution and plastic damage near the wellbore in soft mudstone formations demonstrate strong thermal sensitivity of wellbore stability with the wellbore mud temperature consistent with that in the practice of drilling engineering.

Assuming that the formation and wellbore mud temperatures are $85^{\circ} \mathrm{C}$ and $45^{\circ} \mathrm{C}$ or $125^{\circ} \mathrm{C}$, respectively, the effective stress changes near the wellbore zone were analyzed. When the wellbore temperature changed from 45 ${ }^{\circ} \mathrm{C}$ to $125^{\circ} \mathrm{C}$, the corresponding temperature increments $\Delta T$ were $-40{ }^{\circ} \mathrm{C}$ and $40{ }^{\circ} \mathrm{C}$, respectively.

Fig.9 shows the effective radial and hoop stress distribution near the wellbore with mud temperature change of $-40^{\circ} \mathrm{C}, 0^{\circ} \mathrm{C}$, and $40^{\circ} \mathrm{C}$ at coupling duration of $24 \mathrm{~h}$. According to the effective radial stress showed in Fig.9 (a), the concentration of the radial compressive stress near the wellbore zone increases with the increase of wellbore temperature. Compared with the wellbore mud temperature 
of $85^{\circ} \mathrm{C}\left(\Delta T=0^{\circ} \mathrm{C}\right)$, the maximum radial compressive stress in the wellbore zone increases by $1.2 \mathrm{MPa}$ at $125^{\circ} \mathrm{C}$ $\left(\Delta T=40^{\circ} \mathrm{C}\right)$. In addition, the corresponding maximum effective hoop compressive stress near the wellbore increases by $8.4 \mathrm{MPa}$ in Fig.9 (b). When the wellbore mud temperature decreases, the effective radial and hoop compressive stresses near the wellbore zone decrease significantly, about $0.27 \mathrm{MPa}$ and $5.5 \mathrm{MPa}$, respectively. The range of temperature effects in soft mudstone formation is approximately 9 times of the wellbore radius $\left(r / r_{w}=9.0\right)$.

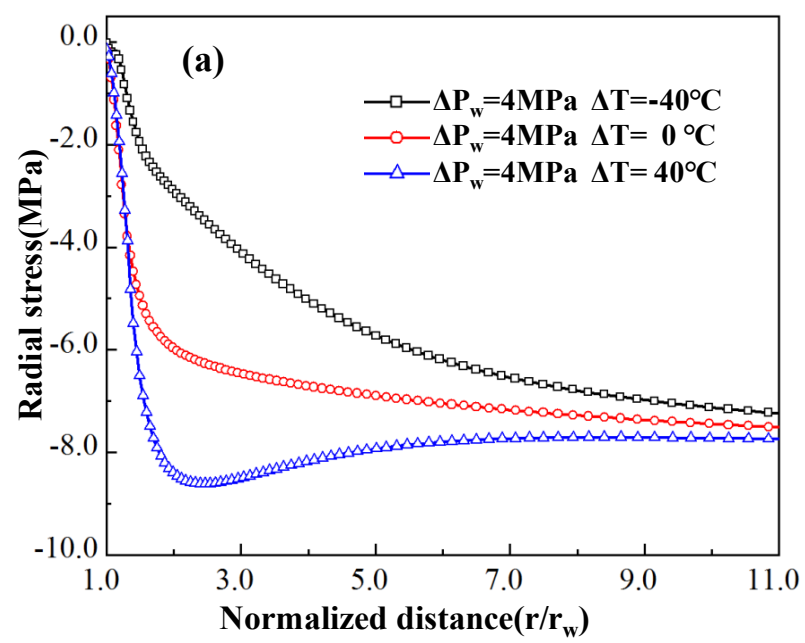

(a) Effective radial stress

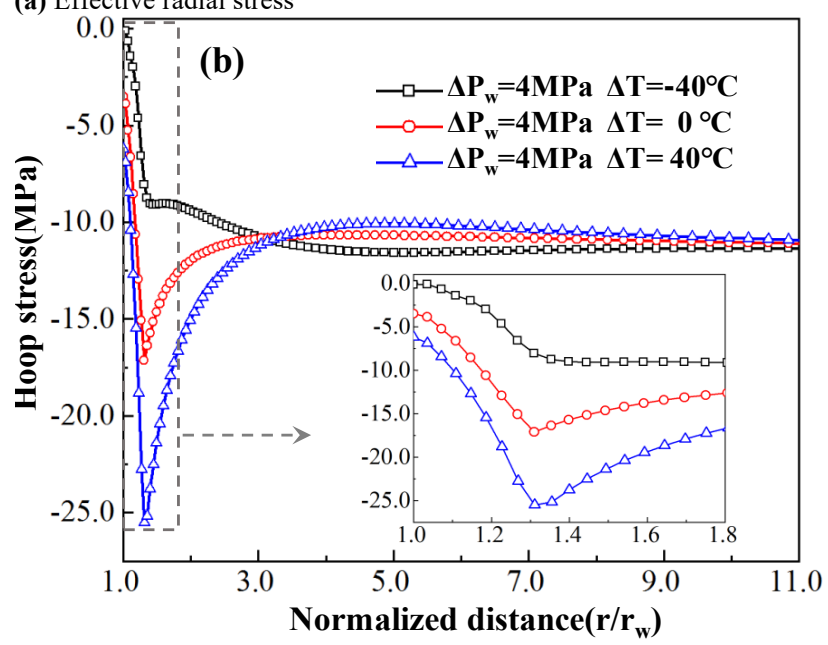

(b) Effective hoop stress

Fig. 9. Effective stress with the normalized distance (at temperature change of $-40,0$, and $40^{\circ} \mathrm{C}$ at coupling duration $24 \mathrm{~h}$ )

Fig. 10 shows the variation of the plastic damage depth near the wellbore zone with the temperature changes. When the wellbore mud temperature was $125^{\circ} \mathrm{C}\left(\Delta T=40{ }^{\circ} \mathrm{C}\right)$, the plastic damage range of wellbore increased from the initial $22.5 \mathrm{~mm}$ to $31.2 \mathrm{~mm}$ after the duration of $24 \mathrm{~h}$. When the wellbore mud temperature was $45{ }^{\circ} \mathrm{C}\left(\Delta T=-40{ }^{\circ} \mathrm{C}\right)$, the effect of compaction occurred near the wellbore zone, and the plastic damage range increased from the initial $18.6 \mathrm{~mm}$ to $26.8 \mathrm{~mm}$. Therefore, a low wellbore mud temperature can effectively inhibit the development of plastic damage near the wellbore zone.

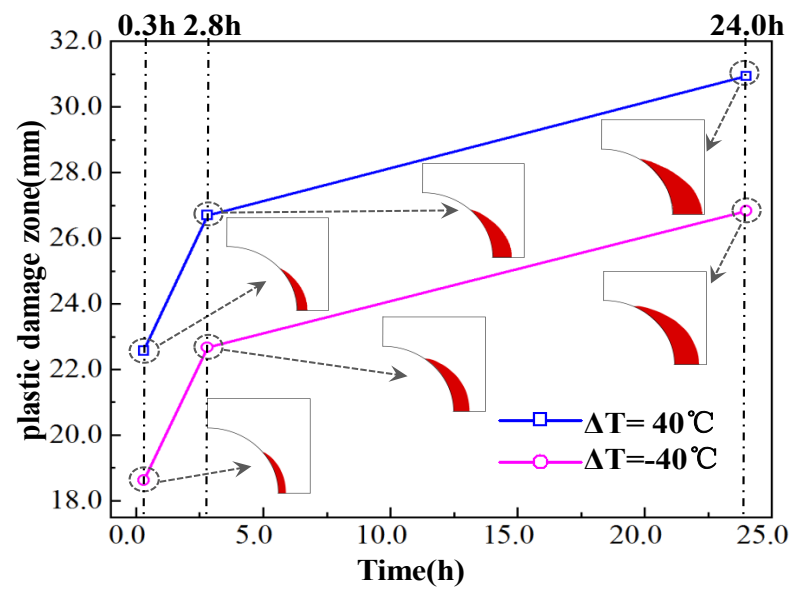

Fig. 10. Effect of temperature load on plastic damage depth

The results of the effective stress and damage near the wellbore zone indicated that the temperature increase in the wellbore mud has a significant negative effect on the stability of the wellbore structure, especially for lowintensity soft mudstone formations. The wellbore mud temperature should be used as the main control parameter for wellbore stability designs.

\section{Conclusions}

To investigate the oil/gas wellbore stability in deep and complicated soft mudstone formations, this study presented a coupled THM model considering various factors, such as the behavior of elastoplastic strain softening, plastic damage, and the drilling process. The following conclusions were drawn from case studies with field data from the Shengli oilfield.

(1) The mechanical characteristics of the wellbore structure in soft mudstone formation is practically controlled by the behavior of wellbore deformation, stress adjustment, and THM coupling effects during drilling.

(2) The localized high permeability zone corresponding to the plastic damage zone plays a key role in wellbore stability, especially in water-absorbing soft mudstone formations. The scale of the high-permeability zone is consistent with that of the plastic damage zone. With the increase in coupling duration, the effective radial compressive stress near the wellbore region is gradually decreased. The maximum hoop effective stress migrates from the wellbore to the periphery, thereby causing the plastic damage zone to expand to 1.3 times that of the wellbore radius at $24 \mathrm{~h}$.

(3) On the basis of the deformation characteristics of soft mudstone, the near-wellbore zone is divided into plastic damage, plastic, and elastic zones from the inside to the outside. The effective radial/hoop stress in the plastic damage zone can be adjusted to the plastic equilibrium state after $0.3 \mathrm{~h}$. This adjustment reduces the local hoop stress concentration of the wellbore.

(4) The temperature of wellbore mud has a significant effect on the stress distribution and damage degree near the wellbore. As the wellbore mud temperature increases by $40{ }^{\circ} \mathrm{C}$, the plastic damage range is enlarged by $2.7 \mathrm{~mm}$. When the temperature decreases by $40{ }^{\circ} \mathrm{C}$, a compacted deformation occurs near the wellbore zone, and the plastic damage range is reduced by $1.7 \mathrm{~mm}$. 
The THM coupling mechanism in this study is significant for the wellbore stability in soft mudstone formations. The proposed model has limitations for the true states of wellbore structures due to the complexity and unpredictability of geological environments. Constructing a
3D wellbore structure model and verifying it with field data will be the emphasis of future studies.

This is an Open Access article distributed under the terms of the Creative Commons Attribution License

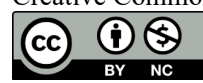

\section{References}

1. Yao J., Huang, Z. Q., Liu W. Z., "Key mechanical problems in the development of deep oil and gas reservoirs". Sci Sin-Phys Mech Astron (in Chinese), 48(4), 2018, pp.5-31.

2. Temitope, A., Ipsita, G., "A review of reactive transport modeling in wellbore integrity problems". Journal of Petroleum Science and Engineering, 175(1), 2019, pp.785-803.

3. Feng, Y., Li, X., Gray, K. E., "An easy-to-implement numerical method for quantifying time-dependent mud cake effects on nearwellbore stresses". Journal of Petroleum Science and Engineering, 164(1), 2018, pp.501-514.

4. Zhang, B., Guan, Z., Lu, N., "Trapped annular pressure caused by thermal expansion in oil and gas wells: A review of prediction approaches, risk assessment and mitigation strategies", Journal of Petroleum Science and Engineering, 172(1), 2019, pp.70-82.

5. Yuan, B., Wood, D. A., "A holistic review of geosystem damage during unconventional oil, gas and geothermal energy recovery", Fuel, 227(1), 2018, pp.99-110.

6. Cui, W., Potts, D. M., "An alternative coupled thermo-hydromechanical finite element formulation for fully saturated soils" Computers and Geotechnics, 94(1), 2018, pp.22-30.

7. Mctigue D. F., "Flow to a heated borehole in porous, thermos-elastic rock: analysis". Water Resources Research, 26, 1990, pp.17631774.

8. Li, J. Y., Li Z, F., "Elastoplastic stress distribution and wellbore stability conditions around wellbore under seepage". Engineering Mechanics (in Chinese), 14(2), 1997, pp.131-137.

9. Roegiers, J. C., "Well modeling: an overview". Oil \& Gas Science and Technology, 57(5), 2002, pp.569-577.

10. Tao, Q., Ghassemi, A., "Poro-thermoelastic borehole stress analysis for determination of the in situ stress and rock strength". Geothermics, 39(3), 2010, pp.250-259.

11. Zhang, W., Sun, J. "Thermo-hydro-mechanical coupling model and comprehensive evaluation method of high temperature geothermal extraction". Progress in Geophysics (in Chinese), 34(2), 2019, pp.0668-0675.
12. Jia, S. P., Wu, B., Chen, W. Z., "Study of thermo-mechano-damage coupling behavior of surrounding rock of deep tunnel". Geotechnical (in Chinese), 35(8), 2014, pp.2375-2384.

13. Kanfar, M. F., Chen, Z., Rahman, S. S., "Fully coupled 3D anisotropic conductive-convective porothermoelasticity modeling for inclined boreholes". Geothermics, 61(1), 2016, pp.135-148.

14. Zhang, P. W., Sun, F. Ye, G. G., "Stability analysis of soft mudshale formation wellbore based on plastic damage theory". Mechanics and Practice (in Chinese), 40(3), 2018, pp.273-280.

15. Li, X., Jaffal, H., Feng, Y., "Wellbore breakouts: Mohr-Coulomb plastic rock deformation, fluid seepage, and time-dependent mudcake buildup". Journal of Natural Gas Science and Engineering, 52(1), 2018, pp.515-528.

16. Wu, Y., Xie, R. J., Liu, S. J., "Wellbore stability law of high temperature and high pressure vertical wells considering temperature effect". Fault-Block Oil \& Gas Field (in Chinese), 26(2), 2019, pp.253-256

17. Wang, J. X., Jiang, A., "Establishment of rock strain softening constitutive model and NR-AL method". Geotechnical (in Chinese), 36(2), 2015, pp.393-402.

18. Xue, S. F., "Theoretical study of Immiscible saturated two-phase flow in nonlinear porous media and applications to petroleum engineering". Beijing: Golden Light Academic Press, China, 2017, pp.26-28.

19. Zhao, X., "Wellbore-Reservoir THM Coupling Model and its Applications for Heavy Oil Thermal Recovery". Doctor thesis of China University of Petroleum, China, 2019, pp.28-33.

20. Liu, J., Li, Q., "Numerical simulation of injection water flow through mudstone interlayer in low permeability oil reservoir development". Disaster Advances, 5(4), 2012, pp.1639-1645.

21. Wang, Y., Papamichos, E., "Conductive heat flow and thermally induced fluid flow around a well bore in a poroelastic medium". Water Resources Research, 30(12), 1994, pp.3375-3384. 